Appl. Set-Valued Anal. Optim. 3 (2021), No. 1, pp. 75-93

Available online at http://asvao.biemdas.com

https://doi.org/10.23952/asvao.3.2021.1.07

\title{
TWO METHODS FOR SOLVING SPLIT COMMON FIXED POINT PROBLEMS OF STRICT PSEUDO-CONTRACTVE MAPPINGS IN HILBERT SPACES WITH APPLICATIONS
}

\author{
A.U. BELLO ${ }^{1,2}$, M.T. OMOJOLA ${ }^{1}$, M.O NNAKWE ${ }^{1, *}$ \\ ${ }^{1}$ Mathematics Institute, African University of Science and Technology, Galadimawa, Abuja, Nigeria \\ ${ }^{2}$ Department of Mathematical Sciences, Federal University, Dutsin-Ma, Katsina State, Nigeria
}

\begin{abstract}
In this paper, we construct Halpern-viscosity and hybrid-projection algorithms. The sequences generated by these algorithms are proved to converge to a common fixed point of two finite families of strictly pseudo-contractive mappings, respectively. Applications of the main convergence theorems are considered. Furthermore, numerical examples are given to show the efficiency of these algorithms.
\end{abstract}

Keywords. Halpern algorithm; Hybrid algorithm; Haugazeau's approach; Strict pseudo-contraction; Viscosity algorithm.

\section{INTRODUCTION}

Let $C$ and $Q$ be nonempty, closed, convex, subsets of real Hilbert spaces $H_{1}$ and $H_{2}$, respectively. The split feasibility problem (SFP) is given by the following:

$$
\text { find } x^{*} \in C \text { such that } A x^{*} \in Q \text {, }
$$

where $A: H_{1} \rightarrow H_{2}$ is a bounded linear map with the adjoint operator $A^{*}: H_{2} \rightarrow H_{1}$. This problem was introduced by Censor and Elfving [1] for the modeling of inverse problems stemming from phase retrievals, image processing and intensity modulated radiation therapy (IMRT) (see, e.g., $[2,3,4])$. The SFP has also been successfully applied in other areas such as immaterial science, computerized tomography, antenna design, sensor networks, data denoising and data compression (see, e.g., [5, 6, 7]). Several iterative algorithms have been introduced for solving SFP, the most notable among them is the CQ method introduced by Byrne [3, 4]. Let $T: H_{1} \rightarrow H_{1}$ and $S: H_{2} \rightarrow H_{2}$ be two mappings with fixed point sets $F(T)$ and $F(S)$, respectively. Then, the split common fixed point problem (SCFPP) is to:

$$
\text { find } x^{*} \in F(T) \text { such that } A x^{*} \in F(S) \text {, }
$$

where $A: H_{1} \rightarrow H_{2}$ is a bounded linear map. This problem originated from Censor and Segal [8] and was studied by employing the following algorithm:

$$
x_{n+1}=T\left(x_{n}-\tau_{n} A^{*}(I-S) A x_{n}\right), \quad n \geq 1,
$$

\footnotetext{
${ }^{*}$ Corresponding author.

E-mail addresses: uabdulmalik@aust.edu.ng (A.U. Bello), momojola@aust.edu.ng (M.T. Omojola), mnnakwe @ aust.edu.ng (M.O Nnakwe).

Received January 30, 2020; Accepted May 7, 2020.
}

(C)2021 Applied Set-Valued Analysis and Optimization 
where $S$ and $T$ are firmly nonexpansive mappings, and $\tau_{n} \in\left(0, \frac{2}{\|A\|^{2}}\right)$ for all $n \geq 1$. The generated sequence was proven to converge weakly to a solution of (1.1). Motivated by the result of Censor and Segal [8], Wang [9] proved a weak convergence theorem for a solution of SCFPP (1.1) given by the following algorithm:

$$
x_{n+1}=x_{n}-\tau_{n}\left[(I-T) x_{n}+A^{*}(I-S) A x_{n}\right], \quad n \geq 1,
$$

where $S$ and $T$ are firmly nonexpansive mappings and $\left\{\tau_{n}\right\} \subset(0, \infty)$ is such that

$$
\sum_{n=1}^{\infty} \tau_{n}=\infty \quad \text { and } \quad \sum_{n=1}^{\infty} \tau_{n}^{2}<\infty .
$$

We observe that the step size $\tau$ in algorithm (1.2) is independent of the norm of the bounded linear operator $A$. Cui and Ceng [10] extended Wang's results to a class of strict pseudo contractions and proved a weak convergence result for approximating solutions of SCFFP.

An important generalization of the SCFPP is the multiple-set splitfeasibility problem (MSSFP) which is to

$$
\text { find } x^{*} \in \cap_{i=1}^{\mathrm{N}} C_{i} \text { such that } A x^{*} \in \cap_{j=1}^{\mathrm{M}} Q_{j},
$$

where $\left\{C_{i}\right\}_{i=1}^{\mathrm{N}}$ and $\left\{Q_{j}\right\}_{j=1}^{\mathrm{M}}$ are nonempty, closed and convex subsets of $H_{1}$ and $H_{2}$, respectively. This problem has been studied by several authors; see, e.g., [11, 12, 13, 14, 15].

Recently, Reich, Tuyen and Trang [16] studied the SCFPP in a more general setting. They formulated the problem as follows:

$$
\text { find } x^{*} \in H_{1} \text { such that } x^{*} \in \Omega:=\cap_{i=1}^{\mathrm{N}} \operatorname{Fix}\left(T_{i}\right) \cap A^{-1}\left(\cap_{j=1}^{\mathrm{M}} \operatorname{Fix}\left(S_{j}\right)\right),
$$

where $\left\{T_{i}\right\}_{i=1}^{\mathrm{N}}: H_{1} \rightarrow H_{1}$ and $\left\{S_{j}\right\}_{j=1}^{\mathrm{M}}: H_{2} \rightarrow H_{2}$ are two finite families of nonexpansive mappings. In fact, they proved the following theorem.

Theorem 1.1. Let $H_{1}$ and $H_{2}$ be two real Hilbert spaces. Let $T_{i}: H_{1} \rightarrow H_{1}, i=1,2,3, \ldots, N$ and $S_{j}: H_{2} \rightarrow H_{2}, j=1,2,3, \ldots, M$, be nonexpansive mappings on $H_{1}$ and $H_{2}$, respectively. Let $A: H_{1} \rightarrow H_{2}$ be a bounded linear operator from $H_{1}$ to $H_{2}$. Suppose that

$$
\Omega:=\cap_{i=1}^{\mathrm{N}} \operatorname{Fix}\left(T_{i}\right) \cap A^{-1}\left(\cap_{j=1}^{\mathrm{M}} \operatorname{Fix}\left(S_{j}\right)\right) \neq \varnothing .
$$

For any $u, x_{0} \in H_{1}$, let $\left\{x_{n}\right\}$ be the sequence generated by

$$
\begin{aligned}
y_{n} & =\sum_{i=1}^{M} a_{i, n} \tilde{T}_{i} x_{n}, \\
z_{n} & =\sum_{j=1}^{N} b_{j, n} S_{j}\left(A y_{n}\right), \\
t_{n} & =y_{n}+\delta A^{*}\left(z_{n}-A y_{n}\right), \\
x_{n+1} & =\alpha_{n} u+\left(1-\alpha_{n}\right) t_{n}, n \geq 0,
\end{aligned}
$$

where $\left\{a_{i, n}\right\}, i=1,2, \ldots, N,\left\{b_{j, n}\right\}, j=1,2, \ldots, M$, and $\left\{\alpha_{n}\right\}$ are positive sequences, $\bar{T}=$ $b_{j, n} I+\left(1-b_{j, n}\right) T$ and $\left\{b_{i, n}\right\} \subset(0,1)$ for each $i=1,2, \ldots, N$ satisfying appropriate conditions. Then, $\left\{x_{n}\right\}$ converges strongly to $x=P_{\Omega}^{H_{1}} u$, where $P_{\Omega}^{H_{1}}$ is the metric projection of $H_{1}$ unto $\Omega$. 
In this paper, we present a strong convergence theorem of solutions of the MSSFP involving strict pseudo-contractive mappings based Halpern-viscosity and hybrid-projection algorithms. Numerical examples are given to show the efficiency of the two algorithms. Our results improve and complement some recent results in the literature.

\section{PRELIMINARIES}

Let $C$ be a nonempty, closed and convex subset of a real Hilbert space $H$. We denote ' $x_{n} \rightarrow x^{*}$, to mean that the sequence $\left\{x_{n}\right\}$ converges weakly to $x^{*}$. We present some definitions and lemmas that will be used in the next section.

Let $P_{C}: H \rightarrow C$ be the metric projection from $H$ onto $C$, that is,

$$
\left\|x-P_{C}(x)\right\| \leq\|x-z\|, \quad \forall z \in C .
$$

Given any $x \in H$ and $z \in C$, the following facts are known:

(i) $z=P_{C}(x) \Longleftrightarrow\langle x-z, y-z\rangle \leq 0, \quad \forall y \in C$;

(ii) $\left\|P_{C} x-y\right\|^{2}+\left\|x-P_{C} x\right\|^{2} \leq\|x-y\|^{2}, \quad \forall y \in C$.

For a space $E$, we say that $E$ has the Kadec Klee property [17] if, for any sequence $\left\{x_{n}\right\}$ in $E$ with $x_{n} \rightarrow x$ and $\left\|x_{n}\right\| \rightarrow\|x\|$, then $\left\|x_{n}-x\right\| \rightarrow 0$ as $n \rightarrow \infty$. It is known that Hilbert spaces have the Kadec Klee property.

Recall that a mapping $T: C \rightarrow C$ is said to be a strict pseudo-contraction if there exists a $\kappa \in[0,1)$ such that

$$
\|T x-T y\|^{2} \leq\|x-y\|^{2}+\kappa\|(I-T) x-(I-T) y\|^{2}, \quad \forall x, y \in C .
$$

If $\kappa=0$ in (2.1), $T$ is said to be nonexpansive. Furthermore, if there exists an $\eta \in(0,1)$ such that

$$
\|T x-T y\| \leq \eta\|x-y\|, \quad \forall x, y \in C,
$$

then $T$ is called a contraction. The following examples show that the class of strict pseudocontractions properly contains the class of nonexpansive mappings.

Example 2.1. Let $T: \mathbb{R} \rightarrow \mathbb{R}$ be a mapping defined by

$$
T x=\left\{\begin{array}{cc}
x, & -\infty<x<0, \\
-\frac{3}{2} x, & 0 \leq x<\infty .
\end{array}\right.
$$

Then, $T$ is $1 / 5$-strictly pseudo-contractive but not nonexpansive.

Example 2.2. Let $T: \ell_{2}(\mathbb{R}) \rightarrow \ell_{2}(\mathbb{R}): x \mapsto-\sqrt{2} x$. Then, $T$ is $1 / 4-$ strictly pseudo-contractive but not nonexpansive.

Lemma 2.1. [18] Let $H$ be a real Hilbert space. Then, we have

(i) $\|x+y\|^{2}=\|x\|^{2}+2\langle x, y\rangle+\|y\|^{2}, \quad \forall x, y \in H$;

(ii) $\|x-y\|^{2} \leq\|y-u\|^{2}+2\langle y-x, u-x\rangle, \quad \forall u, x, y \in H$;

(iii) $\|\alpha x+(1-\alpha) y\|^{2}=\alpha\|x\|^{2}+(1-\alpha)\|y\|^{2}-\alpha(1-\alpha)\|x-y\|^{2}, \quad \forall x, y \in H, \alpha \in[0,1]$;

(iv) $\left\|\sum_{i=1}^{\mathrm{N}} \beta_{i} x_{i}\right\|^{2}=\sum_{i=1}^{\mathrm{N}} \beta_{i}\left\|x_{i}\right\|^{2}-\frac{1}{2} \sum_{i, j=1}^{\mathrm{N}} \beta_{i} \beta_{j}\left\|x_{i}-x_{j}\right\|^{2}, \forall\left\{x_{i}\right\}_{i}^{\mathrm{N}} \subset H,\left\{\beta_{i}\right\}_{i}^{\mathrm{N}} \subset[0,1], N \geq$ 2, where $\sum_{i=1}^{\mathrm{N}} \beta_{i}=1$.

Lemma 2.2. [19] Let $T: C \rightarrow C$ be a strict pseudo-contraction. 
i) Then, $T$ is Lipschitz, that is,

$$
\|T x-T y\| \leq \frac{1+\kappa}{1-\kappa}\|x-y\|, \quad \forall x, y \in C .
$$

ii) $I-T$ is demiclosed at 0 , that is, for any sequence $\left\{x_{n}\right\}$ in $C$ such that $x_{n} \rightarrow \tilde{x}$ and $(I-T) x_{n} \rightarrow 0$, then $(I-T) \tilde{x}=0$.

Lemma 2.3. [20] Let $H$ be a Hilbert space and $C$ a closed, convex, and nonempty subset of $H$. Let $T: C \rightarrow C$ be an $\eta$-strict pseudo-contraction. Define $S x=\zeta x+(1-\zeta)$ Tx for all $x \in C$, where $\eta<\zeta<1$. Then $F(S)=F(T)$.

Lemma 2.4. [21] Let $\left\{s_{n}\right\}$ be a real sequence which does not decrease at infinity in the sense that there exists a subsequence $\left\{s_{n_{k}}\right\}$ such that $s_{n_{k}} \leq s_{n_{k}+1}, \forall k \geq 0$. Define an integer sequence $\{\tau(n)\}$, where $n>n_{0}$, by $\tau(n):=\max \left\{n_{0} \leq k \leq n: s_{k}<s_{k+1}\right\}$. Then, $\tau(n) \rightarrow \infty$ as $n \rightarrow \infty$, and for all $n>n_{0}$, we have $\max \left\{s_{\tau(n)}, s_{n}\right\} \leq s_{\tau(n)+1}$.

Lemma 2.5. [22] Let $\left\{s_{n}\right\}$ be a sequence of nonnegative real numbers satisfying the inequality: $s_{n+1} \leq\left(1-\gamma_{n}\right) s_{n}+\gamma_{n} \delta_{n}, \forall n \geq 0$, where $\left\{\gamma_{n}\right\}_{n \in \mathbb{N}} \subset(0,1)$ and $\left\{\delta_{n}\right\}_{n \in \mathbb{N}} \subset \mathbb{R}$ such that (i) $\sum_{n=0}^{\infty} \gamma_{n}=\infty$, (ii) $\limsup _{n \rightarrow \infty} \delta_{n} \leq 0$. Then, $s_{n} \rightarrow 0$.

\section{MAin RESUlTS}

Theorem 3.1. Let $H_{1}$ and $H_{2}$ be real Hilbert spaces. Let $\left\{T_{i}\right\}_{i=1}^{\mathrm{N}}: H_{1} \rightarrow H_{1}$ and $\left\{S_{j}\right\}_{j=1}^{\mathrm{M}}: H_{2} \rightarrow$ $H_{2}$ be $\left\{\kappa_{i}\right\}_{i=1}^{\mathrm{N}}$ and $\left\{\lambda_{j}\right\}_{j=1}^{\mathrm{M}}$ strict pseudo-contractions, respectively. Suppose that $F: H_{1} \rightarrow H_{1}$ $F$ is an $\eta$-contraction and $\Omega:=\cap_{i=1}^{\mathrm{N}} \operatorname{Fix}\left(T_{i}\right) \cap A^{-1}\left(\cap_{j=1}^{\mathrm{M}} \operatorname{Fix}\left(S_{j}\right)\right) \neq \varnothing$, where $A: H_{1} \rightarrow H_{2}$ is a bounded linear operator, $A \neq 0$, and $A^{*}: H_{2} \rightarrow H_{1}$ is its adjoint operator. For any $x_{0} \in H_{1}$, define the sequence $\left\{x_{n}\right\}$ iteratively by

$$
\left\{\begin{array}{l}
y_{n}=\sum_{i=1}^{\mathrm{N}} a_{i, n} \bar{T}_{i, n} x_{n} \\
z_{n}=\sum_{j=1}^{\mathrm{M}} b_{j, n} \bar{S}_{j, n}\left(A y_{n}\right) \\
t_{n}=y_{n}+\delta A^{*}\left(z_{n}-A y_{n}\right) \\
x_{n+1}=\alpha_{n} F\left(x_{n}\right)+\left(1-\alpha_{n}\right) t_{n}, \quad n \geq 0
\end{array}\right.
$$

where $\kappa=\max \left\{\kappa_{i}\right\}$ and $\lambda=\max \left\{\lambda_{j}\right\}$ with $\kappa<\beta_{i, n}<d<1$ and $\lambda<\gamma_{j, n}<d<1$ for all $i=$ $1,2, \ldots, N, j=1,2, \ldots, M$, and $n \geq 0$. Suppose that $\bar{T}_{i, n}=\beta_{i, n} I+\left(1-\beta_{i, n}\right) T_{i}, \bar{S}_{j, n}=\gamma_{j, n} I+(1-$ $\left.\gamma_{j, n}\right) S_{j}$. Assume that the following conditions hold:

C1) $\delta \in\left(0, \frac{1-\lambda}{\|A\|^{2}}\right), \underline{\lim _{n \rightarrow \infty}}\left(\beta_{i, n}-\kappa\right)>0$ for all $i=1,2, \ldots, N$;

C2) $\left\{\alpha_{n}\right\} \subset(0,1), \lim _{n \rightarrow \infty} \alpha_{n}=0, \sum_{n=0}^{\infty} \alpha_{n}=\infty, \sum_{i=1}^{\mathrm{N}} a_{i, n}=\sum_{j=1}^{\mathrm{M}} b_{j, n}=1$;

C3) $\left\{a_{i, n}\right\}_{i=1}^{\mathrm{N}},\left\{b_{j, n}\right\}_{j=1}^{\mathrm{M}} \subset[a, b] \subset(0,1)$.

Then, the sequence $\left\{x_{n}\right\}$ converges strongly to $x^{\dagger}=P_{S}^{H_{1}} F\left(x^{\dagger}\right)$.

Proof. Let $x, y \in \Omega$ be arbitrarily fixed. Using the fact that $F$ is a contraction and $P_{S}$ is nonexpansive, one has

$$
\left\|P_{S} F(x)-P_{S} F(y)\right\| \leq\|F(x)-F(y)\| \leq \eta\|x-y\| .
$$


Thus, $P_{S} F$ is an $\eta$-contraction on $S$. By using the Banach contraction principle, there exists a unique $x^{\dagger} \in S$ such that $x^{\dagger}=P_{S} F\left(x^{\dagger}\right)$. Note that

$$
\left\|F\left(x_{n}\right)-x^{\dagger}\right\| \leq\left\|F\left(x_{n}\right)-F\left(x^{\dagger}\right)\right\|+\left\|F\left(x^{\dagger}\right)-x^{\dagger}\right\| \leq \eta\left\|t_{n}-x^{\dagger}\right\|+\left\|F\left(x^{\dagger}\right)-x^{\dagger}\right\| .
$$

From Lemma 2.1 (iv) and the definition of strict pseudo-contractions, one has

$$
\begin{aligned}
\left\|y_{n}-x^{\dagger}\right\|^{2}= & \sum_{i=1}^{\mathrm{N}} a_{i, n}\left\|T_{i} x_{n}-T_{i} x^{\dagger}\right\|^{2}-\frac{1}{2} \sum_{i, k=1}^{\mathrm{N}} a_{i, n} a_{k, n}\left\|T_{i} x_{n}-\bar{T}_{k} x_{n}\right\|^{2} \\
\leq & \sum_{i=1}^{\mathrm{N}} a_{i, n}\left\|T_{i} x_{n}-T_{i} x^{\dagger}\right\|^{2} \\
= & \sum_{i=1}^{\mathrm{N}} a_{i, n}\left[\beta_{i, n}\left\|x_{n}-x^{\dagger}\right\|^{2}+\left(1-\beta_{i, n}\right)\left\|T_{i} x_{n}-T_{i} x^{\dagger}\right\|^{2}-\beta_{i, n}\left(1-\beta_{i, n}\right)\left\|x_{n}-T_{i} x_{n}\right\|^{2}\right] \\
\leq & \sum_{i=1}^{\mathrm{N}} a_{i, n}\left[\beta_{i, n}\left\|x_{n}-x^{\dagger}\right\|^{2}+\left(1-\beta_{i, n}\right)\left\|x_{n}-x^{\dagger}\right\|^{2}+\left(1-\beta_{i, n}\right) \kappa\left\|x_{n}-T_{i} x_{n}\right\|^{2}\right. \\
& \left.-\beta_{i, n}\left(1-\beta_{i, n}\right)\left\|x_{n}-T_{i} x_{n}\right\|^{2}\right] \\
= & \left\|x_{n}-x^{\dagger}\right\|^{2}-\sum_{i=1}^{\mathrm{N}} a_{i, n}\left(\beta_{i, n}-\kappa\right)\left(1-\beta_{i, n}\right)\left\|x_{n}-T_{i} x_{n}\right\|^{2} .
\end{aligned}
$$

Similarly, with $A x^{\dagger}=S_{j} A x^{\dagger}$, for all $j=1,2, \ldots, M$, we have

$$
\begin{aligned}
\left\|z_{n}-A x^{\dagger}\right\|^{2} \leq & \sum_{j=1}^{\mathrm{M}} b_{j, n}\left[\gamma_{j, n}\left\|A y_{n}-A x^{\dagger}\right\|^{2}+\left(1-\gamma_{j, n}\right)\left\|A y_{n}-A x^{\dagger}\right\|^{2}\right. \\
& \left.+\left(1-\gamma_{j, n}\right) \lambda\left\|A y_{n}-S_{j}\left(A y_{n}\right)\right\|^{2}-\gamma_{j, n}\left(1-\gamma_{j, n}\right)\left\|A y_{n}-S_{j}\left(A y_{n}\right)\right\|^{2}\right] \\
\leq & \|A\|^{2}\left\|y_{n}-x^{\dagger}\right\|^{2}-\sum_{j=1}^{\mathrm{M}} b_{j, n}\left(\gamma_{j, n}-\lambda\right)\left(1-\gamma_{j, n}\right)\left\|A y_{n}-S_{j}\left(A y_{n}\right)\right\|^{2} .
\end{aligned}
$$

Furthermore, we obtain

$$
\begin{aligned}
\left\|t_{n}-x^{\dagger}\right\|^{2} & =\left\|y_{n}-x^{\dagger}+\delta A^{*}\left(z_{n}-A y_{n}\right)\right\|^{2} \\
& \leq\left\|y_{n}-x^{\dagger}\right\|^{2}+2 \delta\left\langle y_{n}-x^{\dagger}, A^{*}\left(z_{n}-A y_{n}\right)\right\rangle+\delta^{2}\|A\|^{2}\left\|z_{n}-A y_{n}\right\|^{2} \\
& \leq\left\|y_{n}-x^{\dagger}\right\|^{2}+2 \delta\left\langle A y_{n}-A x^{\dagger}, z_{n}-A y_{n}\right\rangle+\delta^{2}\|A\|^{2}\left\|z_{n}-A y_{n}\right\|^{2} .
\end{aligned}
$$

Again, by using Lemma 2.1 (i), we find

$$
\begin{aligned}
\left\langle z_{n}-A y_{n}, A y_{n}-A x^{\dagger}\right\rangle= & \sum_{j=1}^{\mathrm{M}} b_{j, n}\left\langle S_{j}\left(A y_{n}\right)-A y_{n}, A y_{n}-A x^{\dagger}\right\rangle \\
= & \frac{1}{2} \sum_{j=1}^{\mathrm{M}} b_{j, n}\left[\left\|S_{j}\left(A y_{n}\right)-S_{j}\left(A x^{\dagger}\right)\right\|^{2}-\left\|S_{j}\left(A y_{n}\right)-A y_{n}\right\|^{2}\right. \\
& \left.-\left\|A y_{n}-A x^{\dagger}\right\|^{2}\right] .
\end{aligned}
$$


Observe that

$$
\begin{aligned}
\left\|S_{j}\left(A y_{n}\right)-S_{j}\left(A x^{\dagger}\right)\right\|^{2}= & \left\|\gamma_{j, n}\left(A y_{n}-A x^{\dagger}\right)+\left(1-\gamma_{j, n}\right)\left(S_{j}\left(A y_{n}\right)-A x^{\dagger}\right)\right\|^{2} \\
= & \gamma_{j, n}\left\|A y_{n}-A x^{\dagger}\right\|^{2}+\left(1-\gamma_{j, n}\right)\left\|S_{j}\left(A y_{n}\right)-A x^{\dagger}\right\|^{2} \\
& -\gamma_{j, n}\left(1-\gamma_{j, n}\right)\left\|A y_{n}-S_{j}\left(A y_{n}\right)\right\|^{2}
\end{aligned}
$$

and

$$
\left\|S_{j}\left(A y_{n}\right)-A y_{n}\right\|^{2}=\left(1-\gamma_{j, n}\right)^{2}\left\|S_{j}\left(A y_{n}\right)-A y_{n}\right\|^{2} .
$$

Substituting these identities in (3.4) and applying the definition of strict pseudo-contractions, we obtain

$$
\left\langle z_{n}-A y_{n}, A y_{n}-A x^{\dagger}\right\rangle \leq-\frac{1}{2} \sum_{j=1}^{\mathrm{M}}(1-\lambda) b_{j, n}\left(1-\gamma_{j, n}\right)\left\|S_{j}\left(A y_{n}\right)-A y_{n}\right\|^{2}
$$

and

$$
\begin{aligned}
\left\|z_{n}-A y_{n}\right\|^{2} & =\left\|\sum_{j=1}^{\mathrm{M}} b_{j, n} S_{j}\left(A y_{n}\right)-A y_{n}\right\|^{2} \\
& \leq \sum_{j=1}^{\mathrm{M}} b_{j, n}\left\|S_{j}\left(A y_{n}\right)-A y_{n}\right\|^{2} \\
& =\sum_{j=1}^{\mathrm{M}} b_{j, n}\left(1-\gamma_{j, n}\right)^{2}\left\|S_{j}\left(A y_{n}\right)-A y_{n}\right\|^{2} .
\end{aligned}
$$

Substituting (3.5) and (3.6) into (3.3), we arrive at

$$
\left\|t_{n}-x^{\dagger}\right\|^{2} \leq\left\|y_{n}-x^{\dagger}\right\|^{2}-\delta\left[(1-\lambda)-\delta\|A\|^{2}\right] \sum_{j=1}^{\mathrm{M}} b_{j, n}\left(1-\gamma_{j, n}\right)\left\|S_{j}\left(A y_{n}\right)-A y_{n}\right\|^{2} \text {. }
$$

Using (3.2) and (3.7), we get that

$$
\begin{aligned}
\left\|x_{n+1}-x^{\dagger}\right\| & \leq \alpha_{n}\left\|F\left(x_{n}\right)-x^{\dagger}\right\|+\left(1-\alpha_{n}\right)\left\|t_{n}-x^{\dagger}\right\| \\
& \leq \alpha_{n}\left\|F\left(x_{n}\right)-F\left(x^{\dagger}\right)\right\|+\alpha_{n}\left\|F\left(x^{\dagger}\right)-x^{\dagger}\right\|+\left(1-\alpha_{n}\right)\left\|x_{n}-p\right\| \\
& \leq\left[1-\alpha_{n}(1-\eta)\right]\left\|x_{n}-x^{\dagger}\right\|+\alpha_{n}\left\|F\left(x^{\dagger}\right)-x^{\dagger}\right\| \\
& \leq \max \left\{\left\|x_{n}-x^{\dagger}\right\|, \frac{\left\|F\left(x^{\dagger}\right)-x^{\dagger}\right\|}{1-\eta}\right\} \\
\vdots & \leq \max \left\{\left\|x_{0}-x^{\dagger}\right\|, \frac{\left\|F\left(x^{\dagger}\right)-x^{\dagger}\right\|}{1-\eta}\right\} .
\end{aligned}
$$

Thus, sequence $\left\{x_{n}\right\}$ is bounded. Consequently, $\left\{y_{n}\right\},\left\{z_{n}\right\},\left\{t_{n}\right\}$, and $\left\{F\left(x_{n}\right)\right\}$ are also bounded, respectively. Note that $x^{\dagger}=P_{S}^{H_{1}} F\left(x^{\dagger}\right)$. From Lemma 2.1 (iii), Condition C2), (3.2), and (3.7), 
we see that

$$
\begin{aligned}
& \left\|x_{n+1}-x^{\dagger}\right\|^{2} \\
& =\alpha_{n}\left\|F\left(x_{n}\right)-x^{\dagger}\right\|^{2}+\left(1-\alpha_{n}\right)\left\|t_{n}-x^{\dagger}\right\|^{2}-\alpha_{n}\left(1-\alpha_{n}\right)\left\|F\left(x_{n}\right)-t_{n}\right\|^{2} \\
& \leq \alpha_{n}\left\|F\left(x_{n}\right)-x^{\dagger}\right\|^{2}+\left\|t_{n}-x^{\dagger}\right\|^{2} \\
& \leq \alpha_{n}\left\|F\left(x_{n}\right)-x^{\dagger}\right\|^{2}+\left\|y_{n}-x^{\dagger}\right\|^{2}-\delta\left(1-\lambda-\delta\|A\|^{2}\right) \sum_{j=1}^{\mathrm{M}} b_{j, n}\left(1-\gamma_{j, n}\right)\left\|S_{j}\left(A y_{n}\right)-A y_{n}\right\|^{2} \\
& \leq \alpha_{n}\left\|F\left(x_{n}\right)-x^{\dagger}\right\|^{2}+\left\|x_{n}-x^{\dagger}\right\|^{2}-\sum_{i=1}^{\mathrm{N}} a_{i, n}\left(\beta_{i, n}-\kappa\right)\left(1-\beta_{i, n}\right)\left\|x_{n}-T_{i} x_{n}\right\|^{2} \\
& \quad-\delta\left(1-\lambda-\delta\|A\|^{2}\right) \sum_{j=1}^{\mathrm{M}} b_{j, n}\left(1-\gamma_{j, n}\right)\left\|S_{j}\left(A y_{n}\right)-A y_{n}\right\|^{2} .
\end{aligned}
$$

Consequently, we have that

$$
\begin{aligned}
& \sum_{i=1}^{\mathrm{N}} a_{i, n}\left(\beta_{i, n}-\kappa\right)\left(1-\beta_{i, n}\right)\left\|x_{n}-T_{i} x_{n}\right\|^{2}+\delta\left(1-\lambda-\delta\|A\|^{2}\right) \sum_{j=1}^{\mathrm{M}} b_{j, n}\left(1-\gamma_{j, n}\right)\left\|S_{j}\left(A y_{n}\right)-A y_{n}\right\|^{2} \\
& \leq \alpha_{n}\left\|F\left(x_{n}\right)-x^{\dagger}\right\|^{2}+\left(\left\|x_{n}-x^{\dagger}\right\|^{2}-\left\|x_{n+1}-x^{\dagger}\right\|^{2}\right) .
\end{aligned}
$$

By Condition C1), we see that there exists $N_{0} \in \mathbb{N}$ and a positive number $\tau$ such that $\left(\beta_{i, n}-\kappa\right)>$ $\tau$, for all $i=1,2, \ldots, N$ and $n \geq 0$. In view of conditions C2) and C3), we obtain

$$
\begin{aligned}
& a \tau(1-d) \sum_{i=1}^{\mathrm{N}}\left\|x_{n}-T_{i} x_{n}\right\|^{2}+a \delta\left(1-\lambda-\delta\|A\|^{2}\right)(1-d) \sum_{j=1}^{\mathrm{M}}\left\|S_{j}\left(A y_{n}\right)-A y_{n}\right\|^{2} \\
& <\alpha_{n}\left\|F\left(x_{n}\right)-x^{\dagger}\right\|^{2}+\left(\left\|x_{n}-x^{\dagger}\right\|^{2}-\left\|x_{n+1}-x^{\dagger}\right\|^{2}\right) .
\end{aligned}
$$

Next, we split the proof into two cases.

Case 1. There exists an $n_{0} \in \mathbb{N}$ such that $\left\{\left\|x_{n}-x^{\dagger}\right\|\right\}_{n \geq n_{0}}$ is nonincreasing. Then, the sequence $\left\{\left\|x_{n}-x^{\dagger}\right\|\right\}_{n \in \mathbb{N}}$ is convergent. Applying C2) and (3.10), we get

$$
\lim _{n \rightarrow \infty}\left\|x_{n}-T_{i} x_{n}\right\|=0 \quad \text { and } \quad \lim _{n \rightarrow \infty}\left\|S_{j}\left(A y_{n}\right)-A y_{n}\right\|=0
$$

for all $i=1,2, \ldots, N$ and $j=1,2, \ldots, M$. From (3.6) and (3.11), we get that $\left\|z_{n}-A y_{n}\right\| \rightarrow 0$ and $\left\|t_{n}-y_{n}\right\| \leq \delta\|A\|\left\|z_{n}-A y_{n}\right\| \rightarrow 0$ as $n \rightarrow \infty$. Using the definition of each $T_{i}$ and condition C3), we have that

$$
\left\|y_{n}-x_{n}\right\|^{2}=\left\|\sum_{i=1}^{\mathrm{N}} a_{i, n}\left(T_{i} x_{n}-x_{n}\right)\right\|^{2} \leq \sum_{i=1}^{\mathrm{N}} a_{i, n}\left(1-\beta_{i, n}\right)^{2}\left\|T_{i} x_{n}-x_{n}\right\|^{2} \rightarrow 0
$$

as $n \rightarrow \infty$. Let $\left\{x_{n_{k}}\right\}$ be a subsequence of $\left\{x_{n}\right\}$ such that

$$
\limsup _{n \rightarrow \infty}\left\langle F\left(x^{\dagger}\right)-x^{\dagger}, x_{n}-x^{\dagger}\right\rangle=\lim _{k \rightarrow \infty}\left\langle F\left(x^{\dagger}\right)-x^{\dagger}, x_{n_{k}}-x^{\dagger}\right\rangle \text {. }
$$


In Lemma 2.1(ii), setting $y=x_{n+1}, x=x^{\dagger}$, and $u=\alpha_{n} F\left(x^{\dagger}\right)+\left(1-\alpha_{n}\right) x^{\dagger}$; we conclude from the convexity of $\|\cdot\|^{2}$ that

$$
\begin{aligned}
\left\|x_{n+1}-x^{\dagger}\right\|^{2} & \leq \| \alpha_{n}\left(F\left(x_{n}\right)-F\left(x^{\dagger}\right)\right)+\left.\left(1-\alpha_{n}\right)\left(t_{n}-x^{\dagger}\right)\right|^{2}+2 \alpha_{n}\left\langle x_{n+1}-x^{\dagger}, F\left(x^{\dagger}\right)-x^{\dagger}\right\rangle \\
& \leq\left[1-\alpha_{n}(1-\eta)\right]\left\|x_{n}-x^{\dagger}\right\|^{2}+2 \alpha_{n}(1-\eta) \frac{\left\langle x_{n+1}-x^{\dagger}, F\left(x^{\dagger}\right)-x^{\dagger}\right\rangle}{1-\eta} .
\end{aligned}
$$

Without loss of generality, we assume that $x_{n_{k}} \rightarrow x^{*}$ as $k \rightarrow \infty$. Using Lemma 2.2ii) and (3.11), we easily see that $x^{*} \in \bigcap_{i=1}^{\mathrm{N}} \operatorname{Fix}\left(T_{i}\right)$. From (3.12), it follows that, for any $y \in H_{1}$,

$$
\left\langle y_{n_{k}}, y\right\rangle=\left\langle y_{n_{k}}-x_{n_{k}}, y\right\rangle+\left\langle x_{n_{k}}, y\right\rangle \rightarrow\left\langle x^{*}, y\right\rangle \quad \text { as } k \rightarrow \infty .
$$

This implies that $y_{n_{k}} \rightarrow x^{*}$. Since $A$ is bounded linear map and $y_{n_{k}} \rightarrow x^{*}$, then, for all $z \in H_{2}$,

$$
\left\langle A y_{n_{k}}, z\right\rangle=\left\langle y_{n_{k}}, A^{*} z\right\rangle \rightarrow\left\langle x^{*}, A^{*} z\right\rangle=\left\langle A x^{*}, z\right\rangle \quad \text { as } k \rightarrow \infty .
$$

Therefore, $A y_{n_{k}} \rightarrow A x^{*}$ as $k \rightarrow \infty$. By Lemma 2.2ii) and (3.11), we obtain $x^{*} \in A^{-1}\left(\cap_{j=1}^{\mathrm{M}} \operatorname{Fix}\left(S_{j}\right)\right)$. So, $x^{*} \in \Omega$. In view of Lemma (2), we have

$$
\lim _{k \rightarrow \infty}\left\langle F\left(x^{\dagger}\right)-x^{\dagger}, x_{n_{k}}-x^{\dagger}\right\rangle=\left\langle F\left(x^{\dagger}\right)-x^{\dagger}, x^{*}-x^{\dagger}\right\rangle \leq 0 .
$$

Employing Lemma 2.5 implies that $\left\{x_{n}\right\}$ converges to $x^{\dagger}=P_{S} F\left(x^{\dagger}\right)$, which completes the proof.

Case 2. Suppose that Case 1 does not hold. Then, there exists a subsequence $\left\{x_{n_{\ell}}\right\}$ of $\left\{x_{n}\right\}$ such that $\left\|x_{n_{\ell}}-x^{\dagger}\right\|<\left\|x_{n_{\ell}+1}-x^{\dagger}\right\|$ for all $l \in\{0\} \cup \mathbb{N}$. Define an integer sequence $\{\tau(n)\}$, where $n>n_{0}$, by

$$
\tau(n):=\max \left\{n_{0} \leq \ell \leq n:\left\|x_{l}-x^{\dagger}\right\|<\left\|x_{l+1}-x^{\dagger}\right\|\right\},
$$

for some $n_{0}$ sufficiently large. Usig Lemma 2.4, we have $\tau(n) \rightarrow \infty$ as $n \rightarrow \infty$, and, for all $n>n_{0}$,

$$
\left\|x_{\tau(n)}-x^{\dagger}\right\|<\left\|x_{\tau(n)+1}-x^{\dagger}\right\| \text {. }
$$

Using similar arguments used in (3.2) and (3.7) as well as the convexity of $\|\cdot\|^{2}$, we arrive at

$$
\begin{aligned}
0 & \leq\left\|x_{\tau(n)+1}-x^{\dagger}\right\|^{2}-\left\|x_{\tau(n)}-x^{\dagger}\right\|^{2} \\
& \leq \alpha_{\tau(n)}\left\|F\left(x_{\tau(n)}\right)-x^{\dagger}\right\|^{2}+\left(1-\alpha_{\tau(n)}\right)\left\|t_{\tau(n)}-x^{\dagger}\right\|^{2}-\left\|x_{\tau(n)}-x^{\dagger}\right\|^{2} \\
& \leq \alpha_{\tau(n)}\left\|F\left(x_{\tau(n)}\right)-x^{\dagger}\right\|^{2}+\left(1-\alpha_{\tau(n)}\right)\left\|x_{\tau(n)}-x^{\dagger}\right\|^{2}-\left\|x_{\tau(n)}-x^{\dagger}\right\|^{2} \\
& \leq \alpha_{\tau(n)}\left\|F\left(x_{\tau(n)}\right)-x^{\dagger}\right\|^{2} .
\end{aligned}
$$

By the boundedness of $\left\{F\left(x_{\tau(n)}\right)\right\}$, there exists an $M>0$ such that

$$
0 \leq\left\|x_{\tau(n)+1}-x^{\dagger}\right\|^{2}-\left\|x_{\tau(n)}-x^{\dagger}\right\|^{2} \leq \alpha_{\tau(n)} M .
$$

Using Condition C2) and letting $n \rightarrow \infty$, we have

$$
\left\|x_{\tau(n)+1}-x^{\dagger}\right\|^{2}-\left\|x_{\tau(n)}-x^{\dagger}\right\|^{2} \rightarrow 0 \quad \text { as } n \rightarrow \infty .
$$

Following similar arguments in (3.2) and (3.11), we conclude from (3.16) and (3.10) that

$$
\lim _{n \rightarrow \infty}\left\|x_{\tau(n)}-T_{i} x_{\tau(n)}\right\|=0 \quad \text { and } \quad \lim _{n \rightarrow \infty}\left\|S_{j}\left(A y_{\tau(n)}\right)-A y_{\tau(n)}\right\|=0
$$


for all $i=1,2, \ldots, N$ and $j=1,2, \ldots, M$. Analogous arguments to those in Case 1 yield

$$
\begin{aligned}
\left\|x_{\tau(n)+1}-x^{\dagger}\right\|^{2} \leq & {\left[1-\alpha_{\tau(n)}(1-\eta)\right]\left\|x_{\tau(n)}-x^{\dagger}\right\|^{2} } \\
& +2 \alpha_{\tau(n)}(1-\eta) \frac{\left\langle x_{\tau(n)+1}-x^{\dagger}, F\left(x^{\dagger}\right)-x^{\dagger}\right\rangle}{1-\eta}
\end{aligned}
$$

and

$$
\limsup _{n \rightarrow \infty}\left\langle F\left(x^{\dagger}\right)-x^{\dagger}, x_{\tau(n)}-x^{\dagger}\right\rangle \leq 0
$$

From Lemma 2.5, we obtain the desired conclusion immediately.

Corollary 3.1. Let $H_{1}$ and $H_{2}$ be real Hilbert spaces. Let $\left\{T_{i}\right\}_{i=1}^{\mathrm{N}}: H_{1} \rightarrow H_{1}$ and $\left\{S_{j}\right\}_{j=1}^{\mathrm{M}}$ : $H_{2} \rightarrow H_{2}$ be $\left\{\kappa_{i}\right\}_{i=1}^{\mathrm{N}}$ and $\left\{\lambda_{j}\right\}_{j=1}^{\mathrm{M}}$ strict pseudo-contractions, respectively. Suppose that $\Omega:=$ $\cap_{i=1}^{\mathrm{N}} \operatorname{Fix}\left(T_{i}\right) \cap A^{-1}\left(\cap_{j=1}^{\mathrm{M}} \operatorname{Fix}\left(S_{j}\right)\right) \neq \varnothing$, where $A: H_{1} \rightarrow H_{2}$ is a bounded linear operator, $A \neq 0$, and $A^{*}: H_{2} \rightarrow H_{1}$ is its adjoint operator. For any $x_{0}, u \in H_{1}$, define the sequence $\left\{x_{n}\right\}$ iteratively by

$$
\left\{\begin{array}{l}
y_{n}=\sum_{i=1}^{\mathrm{N}} a_{i, n} \bar{T}_{i, n} x_{n}, \\
z_{n}=\sum_{j=1}^{\mathrm{M}} b_{j, n} \bar{S}_{j, n}\left(A y_{n}\right), \\
t_{n}=y_{n}+\delta A^{*}\left(z_{n}-A y_{n}\right), \\
x_{n+1}=\alpha_{n} u+\left(1-\alpha_{n}\right) t_{n}, \quad n \geq 0,
\end{array}\right.
$$

where $\kappa=\max \left\{\kappa_{i}\right\}$ and $\lambda=\max \left\{\lambda_{j}\right\}$ with $\kappa<\beta_{i, n}<d<1$ and $\lambda<\gamma_{j, n}<d<1$ for all $i=$ $1,2, \ldots, N, j=1,2, \ldots, M$, and $n \geq 0$. Suppose that $\bar{T}_{i, n}=\beta_{i, n} I+\left(1-\beta_{i, n}\right) T_{i}, \bar{S}_{j, n}=\gamma_{j, n} I+(1-$ $\left.\gamma_{j, n}\right) S_{j}$. Assume that the following conditions hold:

C1) $\delta \in\left(0, \frac{1-\lambda}{\|A\|^{2}}\right), \varliminf_{n \rightarrow \infty}\left(\beta_{i, n}-\kappa\right)>0$ for all $i=1,2, \ldots, N$;

C2) $\left\{\alpha_{n}\right\} \subset(0,1), \lim _{n \rightarrow \infty} \alpha_{n}=0, \sum_{n=0}^{\infty} \alpha_{n}=\infty, \sum_{i=1}^{\mathrm{N}} a_{i, n}=\sum_{j=1}^{\mathrm{M}} b_{j, n}=1$;

C3) $\left\{a_{i, n}\right\}_{i=1}^{\mathrm{N}},\left\{b_{j, n}\right\}_{j=1}^{\mathrm{M}} \subset[a, b] \subset(0,1)$.

Then, sequence $\left\{x_{n}\right\}$ converges strongly to $x^{\dagger}=P_{S}^{H_{1}}(u)$.

Proof. The proof follows by employing similar arguments to those in the proof of Theorem 3.1 when $F\left(x_{n}\right)=u$, for all $n \geq 0$.

Theorem 3.2. Let $H_{1}$ and $H_{2}$ be real Hilbert spaces. Let $\left\{T_{i}\right\}_{i=1}^{\mathrm{N}}: H_{1} \rightarrow H_{1}$ and $\left\{S_{j}\right\}_{j=1}^{\mathrm{M}}$ : $H_{2} \rightarrow H_{2}$ be $\left\{\kappa_{i}\right\}_{i=1}^{\mathrm{N}}$ and $\left\{\lambda_{j}\right\}_{j=1}^{\mathrm{M}}$ strict pseudo-contractions, respectively. Suppose that $\Omega:=$ $\cap_{i=1}^{\mathrm{N}} \operatorname{Fix}\left(T_{i}\right) \cap A^{-1}\left(\cap_{j=1}^{\mathrm{M}} \operatorname{Fix}\left(S_{j}\right)\right) \neq \varnothing$, where $A: H_{1} \rightarrow H_{2}$ is a bounded linear operator, $A \neq 0$, and $A^{*}: H_{2} \rightarrow H_{1}$ is its adjoint operator. For any $x_{0}, u \in H_{1}$, define the sequence $\left\{x_{n}\right\}$ iteratively 
by

$$
\left\{\begin{array}{l}
y_{n}=\sum_{i=1}^{\mathrm{N}} a_{i, n} \bar{T}_{i, n} x_{n}, \\
z_{n}=\sum_{j=1}^{\mathrm{M}} b_{j, n} \bar{S}_{j, n}\left(A y_{n}\right), \\
t_{n}=y_{n}+\delta A^{*}\left(z_{n}-A y_{n}\right), \\
w_{n}=\alpha_{n} x_{n}+\left(1-\alpha_{n}\right) t_{n}, \\
C_{n}=\left\{z \in H_{1}:\left\|w_{n}-z\right\| \leq\left\|x_{n}-z\right\|\right\}, \\
Q_{n}=\left\{z \in H_{1}:\left\langle x_{0}-x_{n}, z-x_{n}\right\rangle \leq 0\right\}, \\
x_{n+1}=P_{C_{n} \cap Q_{n}}^{H_{1}}\left(x_{0}\right), \quad n \geq 0,
\end{array}\right.
$$

where $C_{1}=Q_{1}=H_{1}$ and the conditions on $\left\{\alpha_{n}\right\}$ in Theorem 3.1 are replaced by

D1) $\left\{\alpha_{n}\right\} \subset[\alpha, \beta] \subset(0,1)$.

Suppose that condions (1), C2), and C3) of Theorem 3.1 are satisfied. Then, $t\left\{x_{n}\right\}$ converges strongly to $x^{\dagger}=P_{\Omega}^{H_{1}} x_{0}$.

Proof. We first show that the algorithm is well defined. Indeed, for each $n \geq 0$, we can rewrite $C_{n}$ as

$$
C_{n}=\left\{z \in H_{1}:\left\langle x_{n}-w_{n}, z\right\rangle \leq \frac{1}{2}\left(\left\|x_{n}\right\|^{2}-\left\|w_{n}\right\|^{2}\right)\right\} .
$$

Clearly, $C_{n}$ and $Q_{n}$ are closed and convex subsets of $H_{1}$. Fixing $p \in \Omega$, we conclude from Lemma 2.3 that $p=\bar{T}_{i, n} p$ and $A p=\bar{S}_{j, n}$. By Condition C1), (3.2), (3.7) and the convexity of $\|\cdot\|$, we have that

$$
\left\|w_{n}-p\right\| \leq \alpha_{n}\left\|x_{n}-p\right\|+\left(1-\alpha_{n}\right)\left\|t_{n}-p\right\| \leq\left\|x_{n}-p\right\|
$$

Hence, $p \in C_{n}$. Consequently, $\Omega \subset C_{n}$ for all $n \geq 0$. Next, by induction, we show that, for all $n \geq 0, \Omega \subset C_{n} \cap Q_{n}$. For $n=0$, we have $\Omega \subset C_{0} \cap Q_{0}=H_{1}$. Now, suppose $\Omega \subset C_{n} \cap Q_{n}$ for some $n \geq 1$. Thus, by our assumption, $\Omega \neq \varnothing$. Thus, $C_{n} \cap Q_{n}$ is non-empty. Since $x_{n+1}=P_{C_{n} \cap Q_{n}}^{H_{1}}\left(x_{0}\right)$, we have $\left\langle x_{0}-x_{n+1}, z-x_{n+1}\right\rangle \leq 0$ for all $z \in C_{n} \cap Q_{n}$. Since $\Omega \subset C_{n} \cap Q_{n}$ and $p \in \Omega$, we obtain $\left\langle x_{0}-x_{n+1}, p-x_{n+1}\right\rangle \leq 0$. This implies that $p \in Q_{n+1}$ and $\Omega \subset C_{n+1} \cap Q_{n+1}$. Consequently, $\Omega \subset$ $C_{n} \cap Q_{n}$ for all $n \geq 0$.

We now show that $\left\{x_{n}\right\}$ is bounded. Let $x^{\dagger}=P_{S}^{H_{1}}\left(x_{0}\right)$. Since $x^{\dagger} \in S \subset Q_{n}$ for all $n \geq 0$, and $x_{n}=P_{Q_{n}}^{H_{1}}\left(x_{0}\right)$, we have that $\left\|x_{n}-x_{0}\right\| \leq\left\|x^{\dagger}-x_{0}\right\|$ for all $n \geq 0$. Hence, $\left\{x_{n}\right\}$ is bounded. Consequently, $\left\{w_{n}\right\},\left\{y_{n}\right\},\left\{z_{n}\right\}$, and $\left\{t_{n}\right\}$ are bounded.

We now show that $\left\{x_{n}\right\}$ converges to $x^{\dagger}$. We have, by definition, $x_{n+1} \in Q_{n}$. Thus, $\left|x_{n}-x_{0}\right| \mid \leq$ $\left\|x_{n+1}-x_{0}\right\|$ This implies that the sequence $\left\{\left\|x_{n}-x_{0}\right\|\right\}$ is non-increasing. Therefore, the limit $\lim _{n \rightarrow \infty}|| x_{n}-x_{0} \|$ exists. Moreover, we have

$$
\left\|x_{n}-x_{n+1}\right\|^{2} \leq\left\|x_{0}-x_{n+1}\right\|^{2}-\left\|x_{0}-x_{n}\right\|^{2} .
$$

As $n \rightarrow \infty$, we obtain that $\left\|x_{n}-x_{n+1}\right\| \rightarrow 0$ and then $\left\|w_{n}-x_{n+1}\right\| \leq\left\|x_{n}-x_{n+1}\right\| \rightarrow 0$. They show that

$$
\left\|x_{n}-w_{n}\right\| \leq\left\|x_{n}-x_{n+1}\right\|+\left\|x_{n+1}-w_{n}\right\| \rightarrow 0 \text { as } n \rightarrow \infty \text {. }
$$


Following similar arguments to those in (3.8) and (3.9) as well as using the convexity of $\|\cdot\|^{2}$ yield

$$
\begin{aligned}
\left\|w_{n}-x^{\dagger}\right\|^{2} \leq & \alpha_{n}\left\|x_{n}-x^{\dagger}\right\|^{2}+\left(1-\alpha_{n}\right)\left\|t_{n}-x^{\dagger}\right\|^{2} \\
\leq & \alpha_{n}\left\|x_{n}-x^{\dagger}\right\|^{2}+\left(1-\alpha_{n}\right)\left\|y_{n}-x^{\dagger}\right\|^{2} \\
& -\delta\left(1-\lambda-\delta\|A\|^{2}\right)\left(1-\alpha_{n}\right) \sum_{j=1}^{\mathrm{M}} b_{j, n}\left(1-\gamma_{j, n}\right)\left\|S_{j}\left(A y_{n}\right)-A y_{n}\right\|^{2} \\
\leq & \alpha_{n}\left\|x_{n}-x^{\dagger}\right\|^{2}+\left(1-\alpha_{n}\right)\left\|x_{n}-x^{\dagger}\right\|^{2} \\
& -\left(1-\alpha_{n}\right) \sum_{i=1}^{\mathrm{N}} a_{i, n}\left(\beta_{i, n}-\kappa\right)\left(1-\beta_{i, n}\right)\left\|x_{n}-T_{i} x_{n}\right\|^{2} \\
& -\delta\left(1-\lambda-\delta\|A\|^{2}\right)\left(1-\alpha_{n}\right) \sum_{j=1}^{\mathrm{M}} b_{j, n}\left(1-\gamma_{j, n}\right)\left\|S_{j}\left(A y_{n}\right)-A y_{n}\right\|^{2} .
\end{aligned}
$$

It follows that

$$
\begin{aligned}
& \sum_{i=1}^{\mathrm{N}} a_{i, n}\left(\beta_{i, n}-\kappa\right)\left(1-\beta_{i, n}\right)\left\|x_{n}-T_{i} x_{n}\right\|^{2}+\delta\left(1-\lambda-\delta\|A\|^{2}\right) \sum_{j=1}^{\mathrm{M}} b_{j, n}\left(1-\gamma_{j, n}\right)\left\|S_{j}\left(A y_{n}\right)-A y_{n}\right\|^{2} \\
& \leq\left(\frac{\left\|x_{n}-x^{\dagger}\right\|^{2}-\left\|w_{n}-x^{\dagger}\right\|^{2}}{1-\alpha_{n}}\right) \\
& \leq\left(\frac{\left\|x_{n}-x^{\dagger}\right\|+\left\|w_{n}-x^{\dagger}\right\|}{1-\alpha_{n}}\right)\left\|x_{n}-w_{n}\right\| .
\end{aligned}
$$

From the boundedness of $\left\{x_{n}\right\}$ and $\left.\left\{w_{n}\right\}, D 1\right)$, and (3.22), we find that

$$
\left\|x_{n}-T_{i} x_{n}\right\| \rightarrow 0 \text { and }\left\|S_{j}\left(A y_{n}\right)-A y_{n}\right\| \rightarrow 0 \text { as } n \rightarrow \infty,
$$

for all $i=1,2, \ldots, N$ and $j=1,2, \ldots, M$. From (3.6) and (3.23), we see that $\left\|z_{n}-A y_{n}\right\|^{2} \rightarrow 0$ as $n \rightarrow \infty$, which together with (3.21) yields that

$$
\left\|t_{n}-y_{n}\right\|^{2} \leq \delta^{2}\|A\|^{2}\left\|z_{n}-A y_{n}\right\|^{2} \rightarrow 0 \text { as } n \rightarrow \infty .
$$

From Lemma 2.1, we obtain that

$$
\begin{aligned}
\left\|w_{n}-x^{\dagger}\right\|^{2} \leq & \alpha_{n}|| x_{n}-x^{\dagger}\left\|^{2}+\left(1-\alpha_{n}\right)\right\| y_{n}-x^{\dagger}\left\|^{2}-\alpha_{n}\left(1-\alpha_{n}\right)\right\| x_{n}-t_{n} \|^{2} \\
& -\delta\left(1-\lambda-\delta\|A\|^{2}\right)\left(1-\alpha_{n}\right) \sum_{j=1}^{\mathrm{M}} b_{j, n}\left(1-\gamma_{j, n}\right)\left\|S_{j}\left(A y_{n}\right)-A y_{n}\right\|^{2} \\
\leq & \left\|x_{n}-x^{\dagger}\right\|^{2}-\alpha_{n}\left(1-\alpha_{n}\right)\left\|x_{n}-t_{n}\right\|^{2} \\
& -\left(1-\alpha_{n}\right) \sum_{i=1}^{\mathrm{N}} a_{i, n}\left(\beta_{i, n}-\kappa\right)\left(1-\beta_{i, n}\right)\left\|x_{n}-T_{i} x_{n}\right\|^{2} \\
& -\delta\left(1-\lambda-\delta\|A\|^{2}\right)\left(1-\alpha_{n}\right) \sum_{j=1}^{\mathrm{M}} b_{j, n}\left(1-\gamma_{j, n}\right)\left\|S_{j}\left(A y_{n}\right)-A y_{n}\right\|^{2} \\
\leq & \left\|x_{n}-x^{\dagger}\right\|^{2}-\alpha_{n}\left(1-\alpha_{n}\right)\left\|x_{n}-t_{n}\right\|^{2} .
\end{aligned}
$$


From Condition D1) and (3.22) as well as the boundedness of $\left\{x_{n}\right\}$ and $\left\{w_{n}\right\}$, we get that

$$
\begin{aligned}
\left\|x_{n}-t_{n}\right\|^{2} & \leq\left[\frac{\left\|x_{n}-x^{\dagger}\right\|^{2}-\left\|w_{n}-x^{\dagger}\right\|^{2}}{\alpha_{n}\left(1-\alpha_{n}\right)}\right] \\
& \leq\left[\frac{\left\|x_{n}-x^{\dagger}\right\|+\left\|w_{n}-x^{\dagger}\right\|}{\alpha_{n}\left(1-\alpha_{n}\right)}\right]\left\|x_{n}-w_{n}\right\| \rightarrow 0 \text { as } n \rightarrow \infty .
\end{aligned}
$$

Therefore, we have

$$
\left\|x_{n}-y_{n}\right\| \leq\left\|x_{n}-t_{n}\right\|+\left\|t_{n}-y_{n}\right\| \rightarrow 0 \text { as } n \rightarrow \infty .
$$

Let $\left\{x_{n_{k}}\right\}$ be a subsequence of $\left\{x_{n}\right\}$ such that $x_{n_{k}} \rightarrow x^{*}$. Using Lemma 2.2ii) and (3.23), we get that $x^{*} \in \cap_{i=1}^{\mathrm{N}} F\left(T_{i}\right)$. From (3.25), it follows that $y_{n_{k}} \rightarrow x^{*}$. Since $A$ is bounded linear map, then $A y_{n_{k}} \rightarrow A x^{*}$ as $k \rightarrow \infty$. Using Lemma 2.2ii) and (3.23), we see that $x^{*} \in A^{-1}\left(\cap_{j=1}^{\mathrm{M}} \operatorname{Fix}\left(S_{j}\right)\right)$. Therefore, $x^{*} \in \Omega$. Hence, $x_{n_{k}}-x_{0} \rightarrow x^{*}-x_{0}$. In view of $x^{\dagger}=P_{\Omega}^{H_{1}}\left(x_{0}\right)$ and $x^{*} \in \Omega$, we conclude from the weak lower semi-continuity of the norm that

$$
\left\|x^{\dagger}-x_{0}\right\| \leq\left\|x^{*}-x_{0}\right\| \leq \underline{\lim }|| x_{n_{k}}-x_{0}\left\|\leq \varlimsup \lim || x_{n_{k}}-x_{0}\right\| \leq\left\|x^{\dagger}-x_{0}\right\| .
$$

This implies that $\left\|x_{n_{k}}-x_{0}\right\| \rightarrow\left\|x^{*}-x_{0}\right\|$ as $k \rightarrow \infty$. Employing this and $x_{n_{k}}-x_{0} \rightarrow x^{*}-x_{0}$, we have, by the Kadec-Klee property of $H$, that $\left\|x_{n_{k}}-x^{*}\right\| \rightarrow 0$ as $k \rightarrow \infty$. Notice that $x_{n_{k}}=P_{Q_{n_{k}}}\left(x_{0}\right)$ and $x^{\dagger} \in Q_{n_{k}}$. Thus,

$$
\begin{aligned}
-\left\|x_{n_{k}}-x^{\dagger}\right\|^{2} & =-\left\langle x^{\dagger}-x_{0}, x^{\dagger}-x_{n_{k}}\right\rangle+\left\langle x_{0}-x_{n_{k}}, x_{n_{k}}-x^{\dagger}\right\rangle \\
& \geq\left\langle x_{0}-x^{\dagger}, x^{\dagger}-x_{n_{k}}\right\rangle .
\end{aligned}
$$

On the other hand, one has $x_{n_{k}}-x^{\dagger} \rightarrow x^{*}-x^{\dagger}$. Using this and the weak lower semi-continuity of $\|\cdot\|^{2}$, we get that

$$
\begin{aligned}
\left\|x^{\dagger}-x^{*}\right\|^{2} & \leq \underline{\lim }\left\|x^{\dagger}-x_{n_{k}}\right\|^{2} \\
& \leq \overline{\lim }\left\|x^{\dagger}-x_{n_{k}}\right\|^{2} \\
& \leq \varlimsup \\
& =\left\|x^{\dagger}-x^{*}\right\|^{2} .
\end{aligned}
$$

This shows that

$$
\left\|x^{\dagger}-x_{n_{k}}\right\|^{2} \rightarrow\left\|x^{\dagger}-x^{*}\right\|^{2} \rightarrow 0 \quad \text { as } k \rightarrow \infty .
$$

From (3.26) and (3.27), we see that

$$
-\left\|x^{\dagger}-x^{*}\right\|^{2} \geq\left\langle x_{0}-x^{\dagger}, x^{\dagger}-x^{*}\right\rangle
$$

Since $x^{*} \in \Omega$ and $x^{\dagger}=P_{\Omega}^{H_{1}}\left(x_{0}\right)$, then

$$
\left\langle x_{0}-x^{\dagger}, x^{\dagger}-x^{*}\right\rangle \geq 0
$$

Plugging (3.29) into (3.28), we have that

$$
-\left\|x^{\dagger}-x^{*}\right\|^{2} \geq 0
$$

Therefore, $x^{\dagger}=x^{*}$ and, $x_{n_{k}} \rightarrow x^{*}$ as $k \rightarrow \infty$. Thus, $x_{n} \rightarrow x^{\dagger}=P_{S}^{H_{1}}\left(x_{0}\right)$. The proof is complete. 


\section{Applications}

4.1. Split minimum point problems. A multivalued mapping $A: \mathscr{D}(A) \subset H \rightarrow 2^{H}$ with domain $\mathscr{D}(A)=\{x \in H: A x \neq \varnothing\}$ and range $\mathscr{R}(A)=\cup\{A x: x \in H\}$ is monotone if, for every $x, y \in$ $H$,

$$
\langle u-v, x-y\rangle \geq 0 \text { for all } u \in A x, v \in A y .
$$

A monotone map $A$ is maximal monotone if its graph $\operatorname{Gr}(A)=\{(x, y) \in E: x \in \mathscr{D}(A), y \in A x\}$ is not properly contained in the graph of any other monotone map. It is known that if $A$ is maximal monotone, then the range $\mathscr{R}(I+\lambda A)=H$, for $\lambda>0$. Consequently, for $\lambda>0$, the resolvent operator is given by $J_{\lambda}=(I+\lambda A)^{-1}$, where $I$ is the identity map on $H$. The operator $J_{\lambda}$ is always single-valued (see, for instance, [23]).

Let $H_{1}$ and $H_{2}$ be real Hilbert spaces. Let $A: H_{1} \rightarrow H_{2}$ be a bounded linear operator, $A \neq 0$, with the adjoint operator $A^{*}: H_{2} \rightarrow H_{1}$. Let $\left\{\mathscr{A}_{i}\right\}_{i=1}^{\mathrm{N}}: H_{1} \rightarrow 2^{H_{1}}$ and $\left\{\mathscr{B}_{j}\right\}_{j=1}^{\mathrm{M}}: H_{2} \rightarrow 2^{H_{2}}$ be maximal monotone operators such that $\Omega:=\cap_{i=1}^{\mathrm{N}} \mathscr{A}_{i=1}^{-1}(0) \cap A^{-1}\left(\cap_{j=1}^{\mathrm{M}} \mathscr{B}_{j=1}^{-1}(0)\right) \neq \varnothing$. The problem under consideration is to find an element $x^{\dagger} \in \Omega$. Several authors have studied this problems, we refer to $[12,24,25,26]$. In Theorems 3.1 and 3.2, for $r>0$, set $T_{i}=J_{r}^{\mathscr{A}_{i}}$ and $S_{j}=J_{r}^{\mathscr{B}_{j}}$, where $J_{r}^{\mathscr{A}_{i}}=\left(I+r \mathscr{A}_{i}\right)^{-1}$ and $J_{r}^{\mathscr{B}_{j}}=\left(I+r \mathscr{B}_{j}\right)^{-1}$ are the resolvent of the operators $\left\{\mathscr{A}_{i}\right\}_{i=1}^{\mathrm{N}}$ and $\left\{\mathscr{B}_{j}\right\}_{j=1}^{\mathrm{M}}$.

It is well known that the resolvents operators are firmly nonexpansive mappings and thus, strictly pseudo-contractive. Hence, under the assumptions of Theorems 3.1 and 3.2, the sequences generated by algorithms $3.1,3.20$ and 3.21 converge to $x^{\dagger} \in \Omega$.

4.2. Split variational inequality problems. Let $C$ and $Q$ be closed and convex subsets of real Hilbert spaces $H_{1}$ and $H_{2}$, respectively. Let $\mathscr{A}, \mathscr{B}: H_{1} \rightarrow H_{1}$ and $\mathscr{B}: H_{2} \rightarrow H_{2}$ be two monotone and hemi-continuous operators and $A: H_{1} \rightarrow H_{2}$ be a bounded linear map. Then, the split variational inequality problem is to find

$$
x^{*} \in C \text { such that }\left\langle v-x^{*}, G x^{*}\right\rangle \geq 0 \text { and }\left\langle q-A x^{*}, G\left(A x^{*}\right)\right\rangle \geq 0, \forall v \in C, q \in Q \text {. }
$$

We denote by $\operatorname{SOL}(\mathscr{C}, \mathscr{A})$ and $\operatorname{SOL}(\mathscr{Q}, \mathscr{B})$ the solutions of the variational inequalities with respect to $\mathscr{A}$ and $\mathscr{B}$.

We now prove the following theorem.

Theorem 4.1. Let $H_{1}$ and $H_{2}$ be real Hilbert spaces, and let $\mathscr{C}$ and $\mathscr{Q}$ be closed, convex subsets of $H_{1}, H_{2}$, respectively. Assume that $F: H_{1} \rightarrow H_{1}$, where $F$ is an $\eta$-contraction. Let $\left\{\mathscr{A}_{i}\right\}_{i=1}^{\mathrm{N}}$ : $\mathscr{C} \rightarrow H_{1}$ and $\left\{\mathscr{B}_{j}\right\}_{j=1}^{\mathrm{M}}: \mathscr{Q} \rightarrow H_{2}$ be two monotone and hemi-continuous operators. Suppose that $\Omega:=\cap_{i=1}^{\mathrm{N}} \operatorname{SOL}\left(C, \mathscr{A}_{i}\right) \cap A^{-1}\left(\cap_{j=1}^{\mathrm{M}} \operatorname{SOL}\left(Q, \mathscr{B}_{j}\right)\right) \neq \varnothing$, where $A: H_{1} \rightarrow H_{2}$ is a bounded linear operator, $A \neq 0$, and $A^{*}: H_{2} \rightarrow H_{1}$ is its adjoint operator. For any $\alpha, \beta>0$ and $x_{0} \in H_{1}$, 
define the sequence $\left\{x_{n}\right\}$ iteratively by

$$
\left\{\begin{array}{l}
y_{n}=\sum_{i=1}^{\mathrm{N}} a_{i, n} \overline{\mathscr{A}}_{i, n}, \\
z_{n}=\sum_{j=1}^{\mathrm{M}} b_{j, n} \overline{\mathscr{B}}_{j, n}, \\
t_{n}=y_{n}+\delta A^{*}\left(z_{n}-A y_{n}\right) \\
x_{n+1}=\alpha_{n} F\left(x_{n}\right)+\left(1-\alpha_{n}\right) t_{n}, \quad n \geq 0
\end{array}\right.
$$

Suppose that $\overline{\mathscr{A}}_{i, n}=\beta_{i, n} I+\left(1-\beta_{i, n}\right) \operatorname{SOL}\left(\mathscr{C}, \alpha \mathscr{A}_{i}+I^{H_{1}}-x_{n}\right), \overline{\mathscr{B}}_{j, n}=\gamma_{j, n} I+\left(1-\gamma_{j, n}\right) \operatorname{SOL}(Q$, $\left.\beta \mathscr{B}_{j}+I^{H_{2}}-A y_{n}\right)$. Assume that the following conditions hold:

C1) $\delta \in\left(0, \frac{1}{\|A\|^{2}}\right),\left\{\alpha_{n}\right\} \subset(0,1), \lim _{n \rightarrow \infty} \alpha_{n}=0, \sum_{n=0}^{\infty} \alpha_{n}=\infty, \sum_{i=1}^{\mathrm{N}} a_{i, n}=\sum_{j=1}^{\mathrm{M}} b_{j, n}=1$;

C2) $\left\{a_{i, n}\right\}_{i=1}^{\mathrm{N}},\left\{b_{j, n}\right\}_{j=1}^{\mathrm{M}} \subset[a, b] \subset(0,1),\left\{\beta_{i, n}\right\}_{i=1}^{\mathrm{N}},\left\{\gamma_{j, n}\right\}_{j=1}^{\mathrm{M}} \subset[c, d] \subset(0,1)$.

Then, $\left\{x_{n}\right\}$ converges strongly to $x^{\dagger}=P_{\Omega}^{H_{1}} F\left(x^{\dagger}\right)$, where $I^{H_{1}}$ and $I^{H_{2}}$ are the identity operators on $\mathrm{H}_{1}$ and $\mathrm{H}_{2}$, respectively.

Proof. Define the following mappings $A_{\mathscr{A}_{i}}: H \rightarrow H \times H$ by

$$
A_{\mathscr{A}_{i}} x= \begin{cases}\mathscr{A}_{i} x+\mathrm{N}_{\mathscr{C}}(x), & x \in \mathscr{C}, \\ \varnothing, & x \notin \mathscr{C},\end{cases}
$$

and $B_{\mathscr{B}_{j}}: H \rightarrow H \times H$ by

$$
B_{\mathscr{B}_{j}} x= \begin{cases}\mathscr{B}_{j} x+\mathrm{N}_{\mathscr{Q}}(x), & x \in \mathscr{Q}, \\ \varnothing, & x \notin \mathscr{Q},\end{cases}
$$

where $\mathrm{N}_{\mathscr{C}}(x)=\left\{y \in H_{1}:\langle z-x, y\rangle\right.$ for all $\left.z \in \mathscr{C}\right\}$ and $\mathrm{N}_{\mathscr{Q}}(x)=\left\{u \in H_{2}:\langle v-x, u\rangle\right.$ for all $\left.v \in \mathscr{Q}\right\}$. According to Rockafellar [27], $A_{\mathscr{A}_{i}}, i=1,2, \ldots, \mathrm{N}$ and $B_{\mathscr{B}_{j}}, j=1,2, \ldots, \mathrm{M}$, are maximal monotone. Observe that

$$
\chi_{n}=\operatorname{SOL}\left(C, \alpha \mathscr{A}_{i}+I^{H_{1}}-x_{n}\right) \quad \text { if and only if }\left\langle z-\chi_{n}, \alpha \mathscr{A}_{i} \chi_{n}+\chi_{n}-x_{n}\right\rangle \geq 0
$$

for all $i=1,2, \ldots, \mathrm{N}$ and $z \in H_{1}$. This implies that $-\alpha \mathscr{A}_{i} \chi_{n}-\chi_{n}+x_{n} \in \alpha \mathrm{N}_{\mathscr{Q}}\left(\chi_{n}\right)$. Consequently, $\chi_{n}=J_{\alpha}^{\mathscr{A}_{i}}\left(x_{n}\right)$. Similarly, if we define $\xi_{n}=\operatorname{SOL}\left(C, \alpha \mathscr{A}_{i}+I^{H_{2}}-x_{n}\right)$, then $\xi_{n}=J_{\beta}^{\mathscr{B}_{j}}\left(A y_{n}\right)$. Since $J_{\alpha}^{\mathscr{A}_{i}}$ and $J_{\beta}^{\mathscr{B}_{j}}, i=1,2, \ldots, \mathrm{N}, j=1,2, \ldots, \mathrm{M}$ are non-expansive mappings, the proof follows that of Theorem 3.1.

\section{NUMERICAL EXAMPLES}

In this section, we present some numerical examples to show the effectiveness of the proposed algorithms. Numerical experiments were carried out on a MATLAB environment on a system with a processor Pentium(R) Dual-Core CPU T4200 @ 2.00GHz, 3.00 GB RAM, and a 64-bit Operating System.

Example 5.1. In this example, we approximate a common fixed point of some strict pseudocontraction mappings. We set $H_{1}=\mathbb{R}^{p}, p \geq 1$, and $H_{2}=\ell_{2}(\mathbb{R})$ with their usual norms. For fixed $\mathrm{N}, \mathrm{M}>1$, set $i=1,2, \ldots, \mathrm{N}$ and $j=1,2, \ldots, \mathrm{M}$, and $n \in \mathbb{N}$. Define a map $T_{i}: \mathbb{R}^{p} \rightarrow \mathbb{R}^{p}$ by $x \mapsto-\frac{3}{2} x$. It is easy to see that $T_{i}$ is a $\kappa:=\frac{1}{5}-$ strict pseudo-contraction map for each 
$i=1,2, \ldots, \mathrm{N}$. Choosing $\beta_{i, n}=\frac{1}{2}$ implies that $\bar{T}_{i, n} x=-\frac{1}{4} x$ for all $i=1,2, \ldots, \mathrm{N}, n \geq 0$, and $x \in \mathbb{R}^{p}$. Setting $a_{i, n}=\frac{2 i}{\mathrm{~N}(\mathrm{~N}+1)}$, we have

$$
\sum_{i=1}^{\mathrm{N}} a_{i, n}=1 \text { and } \sum_{i=1}^{\mathrm{N}} a_{i, n} \bar{T}_{i, n} x=-\frac{x}{4} \text { for all } x \in \mathbb{R}^{p} .
$$

Moreover, it is easy to see that $\cap_{i=1}^{\mathrm{N}} \operatorname{Fix}\left(T_{i}\right)=\cap_{i=1}^{\mathrm{N}} \operatorname{Fix}\left(\bar{T}_{i, n}\right)=\{0\}$. Now, define a map $A: \mathbb{R}^{p} \rightarrow$ $\ell_{2}(\mathbb{R})$ such that

$$
x=\left\{x_{k}\right\}_{k=1}^{p} \mapsto A x=\left\{A_{k} x\right\}_{k=1}^{\infty}: A_{k} x= \begin{cases}2 x_{k}, & 1 \leq k \leq p, \\ 0, & \text { otherwise } .\end{cases}
$$

Obviously, the map $A$ is bounded and linear with $\|A\|=2$. Define also the following map: $S_{j}: \ell_{2}(\mathbb{R}) \rightarrow \ell_{2}(\mathbb{R})$ by $S_{j} y=-\frac{5}{2} y$ for all $y \in \ell_{2}(\mathbb{R})$. It is clear that $S_{j}$ is a $\lambda:=\frac{29}{49}-$ strict pseudo-contraction map. Setting $\gamma_{j, n}=\frac{2}{3}$ implies that $\bar{S}_{j, n} y=-\frac{1}{6} y$ for all $j=1,2, \ldots, \mathrm{M}, n \geq 0$, $y \in \ell_{2}(\mathbb{R})$. Taking $b_{j, n}=\frac{2 j}{\mathrm{M}(\mathrm{M}+1)}$, we obtain that

$$
\sum_{j=1}^{\mathrm{M}} b_{j, n}=1 \text { and } \sum_{j=1}^{\mathrm{M}} b_{j, n} \bar{S}_{j, n} y=-\frac{y}{6} \text { for all } y \in \ell_{2}(\mathbb{R}) .
$$

Let $x \in \mathbb{R}^{p}$ and $y \in \ell_{2}(\mathbb{R})$. Then, from the definition of $A$ in (5.1), we have that

$$
\begin{aligned}
\langle A x, y\rangle_{\ell_{2}(\mathbb{R})} & =2\left\langle\left(x^{1}, x^{2}, \cdots, x^{p}, 0,0, \cdots\right),\left(y^{1}, y^{2}, \cdots, y^{p}, \cdots\right)\right\rangle_{\ell_{2}(\mathbb{R})} \\
& =\left\langle\left(x^{1}, x^{2}, \cdots, x^{p}\right), 2\left(y^{1}, y^{2}, \cdots, y^{p}\right)\right\rangle_{\mathbb{R}^{p}} .
\end{aligned}
$$

Therefore, the adjoint operator of $A$ is the map $A^{*}: \ell_{2}(\mathbb{R}) \rightarrow \mathbb{R}^{p}$ by $A^{*}(y)=2\left(y^{1}, y^{2}, \cdots, y^{p}\right)$ for all $y=\left\{y_{k}\right\}_{k=1}^{\infty} \in \ell_{2}(\mathbb{R})$.

It is clear that $\Omega:=\cap_{i=1}^{\mathrm{N}} \operatorname{Fix}\left(T_{i}\right) \cap A^{-1}\left(\cap_{j=1}^{\mathrm{M}} \operatorname{Fix}\left(S_{j}\right)\right) \neq \varnothing$ since $0 \in S$. For computational purposes, we choose $\delta=\frac{1}{2\|A\|^{2}}(1-\lambda)$ and $x_{1}=(1,1, \cdots, 1) \in \mathbb{R}^{p}$. In Algorithm (3.20), we set $u=(1,1, \cdots, 1)$ and define $F: \mathbb{R}^{p} \rightarrow \mathbb{R}^{p}$ by $F(x)=\frac{1}{3} x$ for all $x \in \mathbb{R}^{p}$. Then, $F$ is a $\frac{1}{3}$-contraction. In Algorithms (3.20) and (3.1), $\alpha_{n}=\frac{1}{n+1}$, while $\zeta_{n}=\frac{3 n}{4 n+1}$ in Algorithm (3.21).

Tables 1-4 contain the test results and time for this experiment. The symbol $\mathrm{N}$ represents the number of iterations, while $\left\|x_{\mathrm{N}}\right\|_{2}$ represents the corresponding 2-norm. It can be deduced from Tables 1 and 2 that, as the number of iterations increase, the three algorithms converge to the common fixed point. However, from Tables 3 and 4, it can be inferred that these algorithms reduce in efficiency as dimensions increase. Also from the aforementioned tables and Figure 1 , it is noticeable that the viscosity algorithm (3.1) performs best among its contemporaries. However, the Halpern scheme (3.20) takes the least computational time. 
TABLE 1. Computational results for Example 5.1 in $\mathbb{R}$.

\begin{tabular}{cccccc}
\hline \multirow{2}{*}{$\mathrm{N}$} & Algorithm (3.20) & & & Algorithm (3.21) & \\
\cline { 2 - 3 } \cline { 5 - 6 } & $\left\|x_{\mathrm{N}}\right\|_{2}$ & Time $(\mathrm{secs})$ & & $\left\|x_{\mathrm{N}}\right\|_{2}$ & Time (secs) \\
\hline 10 & $8.0000 \times 10^{-2}$ & 0.000026 & & $1.5573 \times 10^{-1}$ & 0.002370 \\
50 & $1.6000 \times 10^{-2}$ & 0.000066 & & $1.5403 \times 10^{-4}$ & 0.003078 \\
100 & $8.0000 \times 10^{-3}$ & 0.000124 & & $3.2480 \times 10^{-8}$ & 0.006308 \\
150 & $5.3333 \times 10^{-3}$ & 0.000164 & & $7.1255 \times 10^{-12}$ & 0.009161 \\
200 & $4.0000 \times 10^{-3}$ & 0.000212 & & $1.5015 \times 10^{-15}$ & 0.012050 \\
\hline
\end{tabular}

TABLE 2. Computational results for Example 5.1 in $\mathbb{R}$.

\begin{tabular}{ccc}
\hline & \multicolumn{2}{c}{ Algorithm $(3.1)$} \\
\cline { 2 - 3 } $\mathrm{N}$ & $\left\|u_{\mathrm{N}}\right\|_{2}$ & Time $($ secs $)$ \\
\hline 10 & $2.4900 \times 10^{-9}$ & 0.000017 \\
50 & $2.1155 \times 10^{-36}$ & 0.000062 \\
100 & $8.9778 \times 10^{-69}$ & 0.000121 \\
150 & $7.7584 \times 10^{-101}$ & 0.000178 \\
200 & $8.9533 \times 10^{-133}$ & 0.000240 \\
\hline
\end{tabular}

TABLE 3. Computational results for Example 5.1 when $\mathrm{N}=100$.

\begin{tabular}{cccccc}
\hline \multirow{2}{*}{$\operatorname{Dim}$} & \multicolumn{1}{c}{ Algorithm $(3.20)$} & & Algorithm $(3.21)$ & \\
\cline { 2 - 3 } \cline { 5 - 6 } & $\left\|x_{\mathrm{N}}\right\|_{2}$ & Time $(\mathrm{secs})$ & & $\left\|x_{\mathrm{N}}\right\|_{2}$ & Time (secs) \\
\hline 1 & $8.0000 \times 10^{-3}$ & 0.000118 & & $3.2480 \times 10^{-8}$ & 0.006067 \\
10 & $2.5298 \times 10^{-2}$ & 0.001446 & & $1.0271 \times 10^{-7}$ & 0.014259 \\
100 & $8.0000 \times 10^{-2}$ & 0.003222 & & $3.2480 \times 10^{-7}$ & 0.021392 \\
1,000 & $2.5298 \times 10^{-1}$ & 0.022713 & & $1.0271 \times 10^{-6}$ & 0.142859 \\
10,000 & $8.0000 \times 10^{-1}$ & 0.440925 & & $3.2480 \times 10^{-6}$ & 1.631194 \\
100,000 & $2.5298 \times 10^{0}$ & 4.595126 & & $1.0271 \times 10^{-5}$ & 18.86650 \\
\hline
\end{tabular}

TABLE 4. Computational results for Example 5.1 when $\mathrm{N}=100$.

\begin{tabular}{ccc}
\hline \multirow{3}{*}{$\operatorname{Dim}$} & Algorithm $(3.21)$ & \\
\cline { 2 - 3 } & $\left\|x_{\mathrm{N}}\right\|_{2}$ & Time $($ secs $)$ \\
\hline 1 & $3.2480 \times 10^{-8}$ & 0.000120 \\
10 & $1.0271 \times 10^{-7}$ & 0.002884 \\
100 & $3.2480 \times 10^{-7}$ & 0.006929 \\
1,000 & $1.0271 \times 10^{-6}$ & 0.056250 \\
10,000 & $3.2480 \times 10^{-6}$ & 0.829133 \\
100,000 & $1.0271 \times 10^{-5}$ & 9.144194 \\
\hline
\end{tabular}



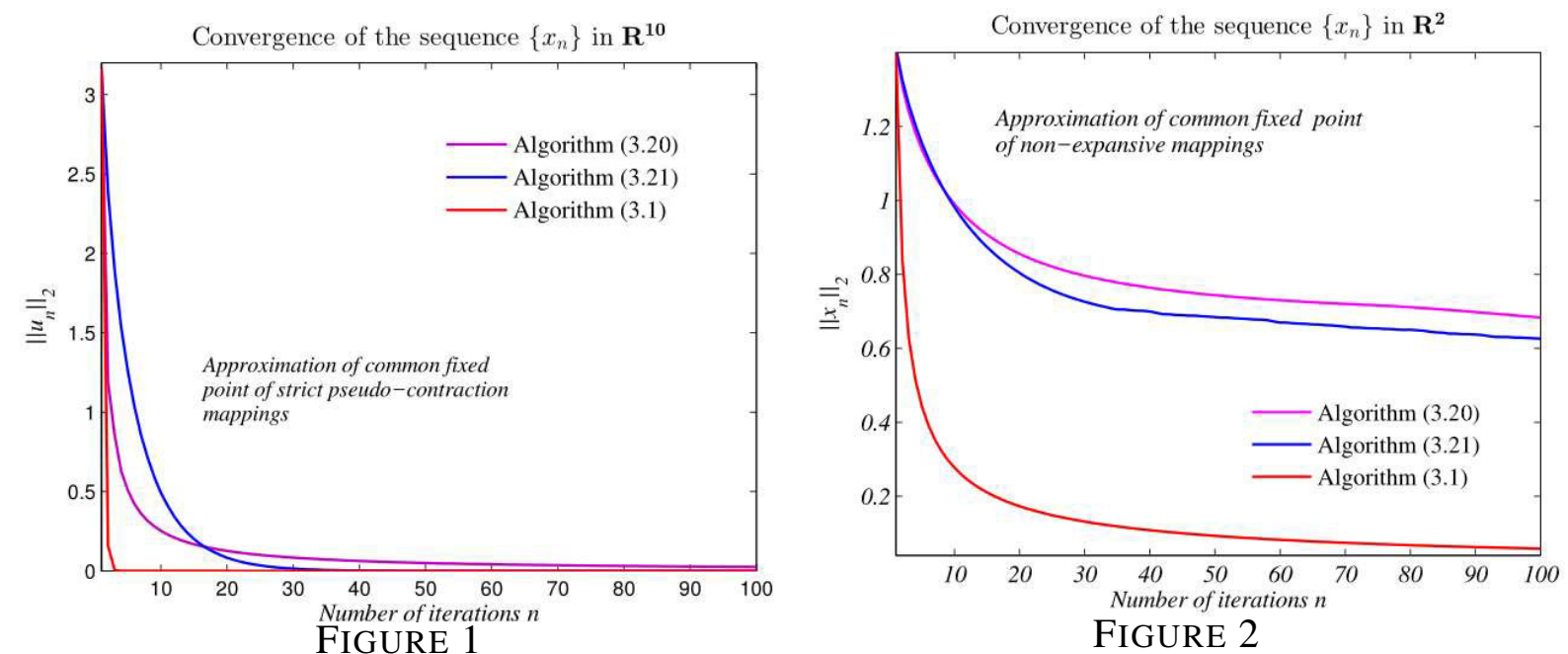

Example 5.2. Let $\mathrm{N}, \mathrm{M}>1$ be arbitrary but fixed. Define the mappings $A, T_{i}, S_{j}: \mathbb{R}^{2} \rightarrow \mathbb{R}^{2}$ by

$$
A u=\left(\frac{1}{2}\left(u_{1}-u_{2}\right), u_{1}-u_{2}\right), \quad A^{*} u=\left(\frac{1}{2}\left(u_{1}+u_{2}\right), u_{2}\right), \quad T_{i} u=\sin u, \quad S_{j} u=P_{B_{r}[a]} u,
$$

for all $u=\left(u_{1}, u_{2}\right) \in \mathbb{R}^{2}, i=1,2, \ldots, \mathrm{N}$, and $j=1,2, \ldots, \mathrm{M}$, where $P_{B_{r}(a)}: \mathbb{R}^{2} \rightarrow B_{r}[a]$ is the metric projection defined by

$$
P_{B_{r}(a)}(v)= \begin{cases}v, & \text { if } v \in B_{r}(a) \\ a+\frac{r}{\|v-a\|}(v-a), & \text { if } v \notin B_{r}(a)\end{cases}
$$

where $B_{r}(a)=\left\{z \in \mathbb{R}^{2}:\|z-a\| \leq r\right\}$. Clearly, $A^{*} u=\left(\frac{1}{2}\left(u_{1}+u_{2}\right), u_{2}\right)$. For computational purposes, we choose $a=0$ and $r=1$. The map $A$ is linear and bounded with $\|A\|=\sqrt{2}$, while $T_{i}$ and $S_{j}$ are both nonexpansive. We set $\delta=\frac{1}{2\|A\|^{2}}, a_{i, n}=\frac{2 i}{\mathrm{~N}(\mathrm{~N}+1)}, b_{j, n}=\frac{2 j}{\mathrm{M}(\mathrm{M}+1)}$, $\beta_{i, n}=\frac{2 n}{3 n+1}$, and $\gamma_{j, n}=\frac{5 n}{8 n+1}$ for each $i$ and $j$ in all the algorithms; $\alpha_{n}=\frac{1}{n+1}$ in Algorithms (3.20) and (3.1). In Algorithm (3.20), $u=(1,1) \in \mathbb{R}^{2}$; in Algorithm (3.1), $F: \mathbb{R}^{2} \rightarrow \mathbb{R}^{2}$ is defined by $F(u)=\frac{1}{3} u$ for all $u \in \mathbb{R}^{2}$ while $\zeta_{n}=\frac{n}{5 n+1}$ in Algorithm (3.21). It is clear that $\Omega:=\cap_{i=1}^{\mathrm{N}} \operatorname{Fix}\left(T_{i}\right) \cap A^{-1}\left(\cap_{j=1}^{\mathrm{M}} \operatorname{Fix}\left(S_{j}\right)\right)=\{0\}$.

Tables 5 and 6 illustrate the computational results and the corresponding test time. It can be observed that, from the those tables and Figure 2, the viscosity algorithm (3.1) converges faster and spends a fairly lesser test period.

\section{CONClusion}

In this paper, we proposed the Halpern, viscosity, and hybrid-type algorithms for estimating the common fixed points of strict pseudo-contraction mappings in Hilbert spaces. The sequences generated by these algorithms were proven to converge strongly. Moreover, we present some applications of our main theorems as well as numerical examples to show the efficiency and accuracy of our algorithms. Furthermore, the hybrid-type algorithm consumed the most time due to the explicit projection's structure provided by the Haugazeau method; while the viscosity approach seems to better estimate the solutions when compared to the other two algorithms. 
TABLE 5. Computational results for Example 5.2 in $\mathbb{R}^{2}$.

\begin{tabular}{cccccc}
\hline \multirow{2}{*}{$\mathrm{N}$} & \multicolumn{1}{c}{ Algorithm (3.20) } & & Algorithm (3.21) & \\
\cline { 2 - 3 } \cline { 5 - 6 } 10 & $9.9018 \times 10^{-1}$ & 0.020598 & & $9.8165 \times 10^{-1}$ & 0.021217 \\
100 & $6.8344 \times 10^{-1}$ & 0.026126 & & $6.2657 \times 10^{-1}$ & 0.037273 \\
1,000 & $3.4863 \times 10^{-1}$ & 0.084964 & & $3.6167 \times 10^{-1}$ & 0.223677 \\
10,000 & $1.6735 \times 10^{-1}$ & 0.879659 & & $1.7800 \times 10^{-1}$ & 2.229821 \\
100,000 & $7.8845 \times 10^{-2}$ & 8.635996 & & $8.4405 \times 10^{-2}$ & 22.24061 \\
\hline
\end{tabular}

TABLE 6. Computational results for Example 5.2 in $\mathbb{R}^{2}$.

\begin{tabular}{ccc}
\hline & Algorithm $(3.1)$ & \\
\cline { 2 - 3 } $\mathrm{N}$ & $\left\|x_{\mathrm{N}}\right\|_{2}$ & Time $($ secs $)$ \\
\hline 10 & $2.7715 \times 10^{-1}$ & 0.011828 \\
100 & $5.8232 \times 10^{-2}$ & 0.022474 \\
1,000 & $1.2371 \times 10^{-2}$ & 0.088361 \\
10,000 & $2.6474 \times 10^{-3}$ & 0.860727 \\
100,000 & $5.6860 \times 10^{-4}$ & 8.519460 \\
\hline
\end{tabular}

\section{Acknowledgments}

The authors are grateful to the referees for the insightful comments and suggestions which improved the contents of this paper.

\section{REFERENCES}

[1] Y. Censor, T. Elfving, A multi projection algorithm using Bregman projections in a product space, Numer. Algo. 8 (1994), 221-239.

[2] A. Taiwo, L.O. Jolaoso, O.T. Mewomo, General alternative regularizationmethod for solving split equality common fixed point problem for quasi-pseudocontractivemappings in Hilbert spaces, Ricerche di Matematica, 69 (2020), 235-259.

[3] Y. Censor, S.Petra, C. Schnorr, Superiorization vs. accelerated convex optimization: The superiorized / regularized least-squares case, J. Appl. Numer. Optim. 2 (2020), 15-62.

[4] C. Byrne, A unified treatment of some iterative algorithms in signal processing and image reconstruction, Inverse Probl. 20 (2004), 103-120.

[5] E. Bonacker, A. Gibali, K.H. Kufer, Nesterov perturbations and projection methods applied to IMRT, J. Nonlinear Var. Anal. 4 (2020), 63-86.

[6] Y. Censor, T. Bortfeld, B. Martin, A. Trofimov, A unified approach for inversion problems in intensitymodulated radiation therapy, Phys. Med. Biol. 51 (2006), 2353-2365.

[7] Y. Shehu, O.S. Iyiola, C.D. Enyi, An iterative algorithm for solving split feasibility problems and fixed point problems in Banach spaces, Numer. Algo. 72 (2016), 835-864.

[8] Y. Censor, A. Segal, The split common fixed point problem for directed operators, J. Convex Anal. 16 (2009), 587-600.

[9] F. Wang, A new method for split common fixed-point problem without priori knowledge of operator norms, J. Fixed Point Theory Appl. 19 (2017), 2427-2436.

[10] H. Cui, L. Ceng, Iterative solutions of the split common fixed point problem for strictly pseudo-contractive mappings, J. Fixed Point Theory Appl. 20 (2018), 92. 
[11] N. Buong, P.T.T. Hoai, K. Thi Binh, Iterative regularization methods for the multiple-sets split feasibility problem in Hilbert spaces, Acta Appl. Math. 165 (2019), 183-197.

[12] S. Suantai, N. Pholasa, P. Cholamjiak, Relaxed CQ algorithms involving the inertial technique for multiplesets split feasibility problems, Revista de La Real Academia de Ciencias Exactas, Fisicas y Naturales. Serie A. Matematicas, 113 (2018), 1081-1099.

[13] X. Wang, Alternating proximal penalization algorithm for the modified multiple-sets split feasibility problems, J. Inequal. Appl. 2018 (2018), 48.

[14] J. Wang, Y. Hu, C.K.W. Yu, X. Zhuang, A family of projection gradient methods for solving the multiple-sets split feasibility problem, J. Optim. Theory Appl. 183 (2019), 520-534.

[15] X. Qin, J.C. Yao, A viscosity iterative method for a split feasibility problem, J. Nonlinear Convex Anal. 20 (2019), 1497-1506.

[16] S. Reich, T.M. Tuyen, N.M. Trang, Parallel iterative methods for solving the split common fixed point problem in Hilbert spaces, Numer. Funct. Anal. Optim. 41 (2020), 778-805.

[17] K. Goebel, W.A. Kirk, Topics in Metric Fixed Point Theory, Cambridge University Press, Cambridge, 1990.

[18] H.H. Bauschke, P.L. Combettes, Convex Analysis and Monotone Operator Theory in Hilbert Spaces, CMS Books in Mathematics. Springer, New York, 2011.

[19] G. Marino, H.K. Xu, Weak and strong convergence theorems for strict pseudocontractions in Hilbert spaces, J. Math. Anal. Appl. 329 (2007), 336-346.

[20] F.E. Browder, W.V. Petryshyn, Construction of fixed points of nonlinear mappings in Hilbert space, J. Math. Anal. Appl. 20 (1967), 197-228.

[21] P.E. Maingé, Strong convergence of projected subgradient methods for nonsmooth and nonstrictly convex minimization, Set-Valued Anal. 16 (2008), 899-912.

[22] H.K. Xu, Strong convergence of an iterative method for nonexpansive and accretive operators, J. Math. Anal. Appl. 314 (2005), 631-643.

[23] F.E. Browder, Nonlinear monotone and accretive operators in Banach spaces, Proc. Natl. Acad. Sci. 61 (1968), 388-393.

[24] S. Reich, T.M. Tuyen, Two projection methods for solving the multiple-set split common null point problem in Hilbert spaces, Optimization, 69 (2020), 1913-1934.

[25] S. Reich, T.M. Tuyen, A new algorithm for solving the split common null point problem in Hilbert spaces, Numer. Algo. 83 (2020), 789-805.

[26] T.M. Tuyen, N.S. Ha, N.T.T. Thuy, A shrinking projection method for solving the split common null point problem in Banach spaces, Numer. Algo. 81 (2019), 813-832.

[27] R.T. Rockafellar, On the maximality of sums of nonlinear monotone operators, Trans. Amer. Math. Soc. 149 (1970), 75-75. 